\title{
Limitations of using synthetic blood clots for measuring in vitro clot capture efficiency of inferior vena cava filters
}

This article was published in the following Dove Press journal:

Medical Devices: Evidence and Research

9 May 2013

Number of times this article has been viewed

\author{
Ronald A Robinson \\ Luke H Herbertson \\ Srilekha Sarkar Das \\ Richard A Malinauskas \\ William F Pritchard \\ Laurence W Grossman \\ Office of Science and Engineering \\ Laboratories, Center for Devices \\ and Radiological Health, US Food \\ and Drug Administration, Silver \\ Spring, MD, USA
}

\begin{abstract}
The purpose of this study was first to evaluate the clot capture efficiency and capture location of six currently-marketed vena cava filters in a physiological venous flow loop, using synthetic polyacrylamide hydrogel clots, which were intended to simulate actual blood clots. After observing a measured anomaly for one of the test filters, we redirected the focus of the study to identify the cause of poor clot capture performance for large synthetic hydrogel clots. We hypothesized that the uncharacteristic low clot capture efficiency observed when testing the outlying filter can be attributed to the inadvertent use of dense, stiff synthetic hydrogel clots, and not as a result of the filter design or filter orientation. To study this issue, sheep blood clots and polyacrylamide (PA) synthetic clots were injected into a mock venous flow loop containing a clinical inferior vena cava (IVC) filter, and their captures were observed. Testing was performed with clots of various diameters $(3.2,4.8$, and $6.4 \mathrm{~mm})$, length-to-diameter ratios $(1: 1,3: 1,10: 1)$, and stiffness. By adjusting the chemical formulation, PA clots were fabricated to be soft, moderately stiff, or stiff with elastic moduli of $805 \pm 2,1696 \pm 10$ and $3295 \pm 37 \mathrm{~Pa}$, respectively. In comparison, the elastic moduli for freshly prepared sheep blood clots were $1690 \pm 360 \mathrm{~Pa}$. The outlying filter had a design that was characterized by peripheral gaps (up to $14 \mathrm{~mm}$ ) between its wire struts. While a low clot capture rate was observed using large, stiff synthetic clots, the filter effectively captured similarly sized sheep blood clots and soft PA clots. Because the stiffer synthetic clots remained straight when approaching the filter in the IVC model flow loop, they were more likely to pass between the peripheral filter struts, while the softer, physiological clots tended to fold and were captured by the filter. These experiments demonstrated that if synthetic clots are used as a surrogate for animal or human blood clots for in vitro evaluation of vena cava filters, the material properties (eg, elastic modulus) and dynamic behavior of the surrogate should first be assessed to ensure that they accurately mimic an actual blood clot within the body.
\end{abstract}

Keywords: blood clot, elastic modulus, polyacrylamide hydrogel, in vitro testing of vena cava filters

\section{Introduction}

Inferior vena cava (IVC) filters are designed to capture thromboemboli and prevent pulmonary embolism in patients who cannot be properly anticoagulated. ${ }^{1}$ For premarket evaluation of IVC filters, the US Food and Drug Administration's Guidance for Cardiovascular Intravascular Filter 510(k) Submissions ${ }^{1}$ recommends that manufacturers test for clot trapping ability and demonstrate that their devices can "capture clinically significant emboli yet still permit sufficient blood flow." Currently, no widely accepted standard testing procedures exist for evaluating clot capture efficiency. ${ }^{2,3}$ In this study, we focused on whether the choice of clot material could affect
Correspondence: Ronald Robinson Center for Devices and Radiological Health, 10903 New Hampshire Ave, WO 62 Room 2120, Silver Spring, MD 20993-0002, USA

$\mathrm{Tel}+\mathrm{I} 30 \mathrm{I} 7962509$

Fax +l 3017969932

Email ronald.robinson@fda.hhs.gov 
the ability of IVC filters to capture clots in an in vitro flow loop test system.

Researchers have studied the clot capture efficiency of IVC filters with a number of different clot materials, including blood clots made from human, bovine, porcine, ovine blood, ${ }^{2,4,5}$ fishing worms molded from soft plastic resin containing dioctyl phthalate, ${ }^{6,7}$ and even cubes of chicken fat. ${ }^{8}$ To evaluate IVC filter performance, Jaeger et al injected synthetic clots cast from $3.5 \%$ polyacrylamide (PA) hydrogels into a flow loop containing a blood-mimicking solution of glycerin and water. ${ }^{9,10}$ Polyacrylamide is a popular and potentially useful test material due to its tunable mechanical properties, but its overall ability to mimic an actual blood clot is not well established. With PA hydrogels, a wide range of physical characteristics can be achieved simply by altering the concentrations of acrylamide or bisacrylamide crosslinker. Jaeger et al reported a PA hydrogel elastic modulus of $800 \mathrm{~Pa}$, which was intended to match the elastic modulus of newly-formed human blood clots., ${ }^{9,10}$

Elasticity refers to the ability of a material to regain its original shape when an applied force is removed. The material behavior of homogeneous isotropic elastic materials is captured by two independent material constants: the elastic (Young's) modulus, E, and Poisson's ratio (v). The elastic modulus is a measure of the material stiffness and is defined as the proportionality constant between uniaxial stress and strain. The elastic modulus is related to shear modulus $(\mathrm{G})$ of the material:

$$
\mathrm{E}=2 \mathrm{G}(1+v)
$$

The dynamic modulus of elasticity $\left(E^{*}\right)$ is used to characterize viscoelastic materials and is composed of storage $\left(\mathrm{E}^{\prime}\right)$ and loss $\left(\mathrm{E}^{\prime \prime}\right)$ components:

$$
\mathrm{E}^{*}=\mathrm{E}^{\prime}+\mathrm{i} \mathrm{E}^{\prime \prime} \text { and } \mathrm{E}^{\prime}=2 \mathrm{G}^{\prime}(1+v)
$$

The storage modulus represents the stored elastic energy, which can be a measure of stiffness. The loss modulus accounts for the viscous properties of the material. The storage modulus of the blood clots or hydrogels can be determined experimentally through compression testing. It should be noted that the physical characteristics of blood clots and synthetic clot materials used in IVC filter capture studies are not usually reported in the literature, perhaps due to the difficulty in performing compression measurements on soft gel-like material.

In this report, we present results from an in vitro clot capture efficiency study, which includes a set of outlying values for one IVC filter. These outliers suggest that under certain conditions the larger PA hydrogel clots are not being captured by the IVC filter, which is of concern since the clinical consequences of larger clots evading capture tend to be more serious than for smaller clots. Because of this discrepancy, we investigated the physical properties of synthetic PA hydrogels relative to those of animal and human blood clots. The goal of this project is not to compare the clot capture efficiencies of different filter designs, but rather to investigate the limitations of using synthetic clots as surrogate blood clots in filter clot capture efficiency experiments and improve the in vitro test methods used to characterize IVC filters. To avoid biasing public opinion with regards to certain filter types, the names of the filters tested in this study have not been disclosed.

\section{Materials and methods Flow loop testing}

To evaluate clot capture efficiency of IVC filters in a flow circuit, a glass flow model of the common iliac veins, iliac bifurcation, and distal IVC with a compliant IVC test section, was developed, which was similar to other reported in vitro testing systems. ${ }^{9,10}$ The in vitro test loop depicted in Figure 1 allowed us to observe the motion and capture of the clot materials under different flow conditions. Two silicone vena cava models with inner diameters of $20 \mathrm{~mm}$ and $28 \mathrm{~mm}$ were used to represent a range of human IVC sizes. ${ }^{4}$ The $15 \mathrm{~cm}$ long, compliant IVC test section was formed by dipping plastic mandrils with flared ends into a castable silicone rubber (Dow Corning 170, Detroit, MI, USA). ${ }^{11}$ The system provided a smooth transition between the glass bifurcation model and the compliant IVC section to minimize flow disturbances at the entrance and exit regions of the test section. Initially, six different IVC filter designs were studied within the model. After each device was deployed in line with the flow, experiments were conducted with the IVC model oriented both vertically and horizontally to investigate potential buoyancy effects of the PA clots. The in vitro flow loop system consisted of two flow configurations: (1) a forward flow loop for studying clot dynamics and clot capture efficiency, and (2) a reverse flow configuration to allow the retrieval of captured clots without disturbing the filter. Ten to thirty PA hydrogel clot injections were performed for each combination of IVC diameter and orientation to study clot capture efficiency, depending on binomial sampling theory. Clot capture efficiency (CCE) is defined as the ratio of the number of clots captured to the number of clots injected. ${ }^{12}$ The test fluid was comprised of $52 \%$ by weight of glycerin in water. The fluid, which was maintained at $37^{\circ} \mathrm{C}$, had a density of $1.12 \mathrm{~g} / \mathrm{cc}$ and a kinematic viscosity of $3.1 \mathrm{cSt}$ to 


\section{Final vena cava filter loop (fwd flow)}

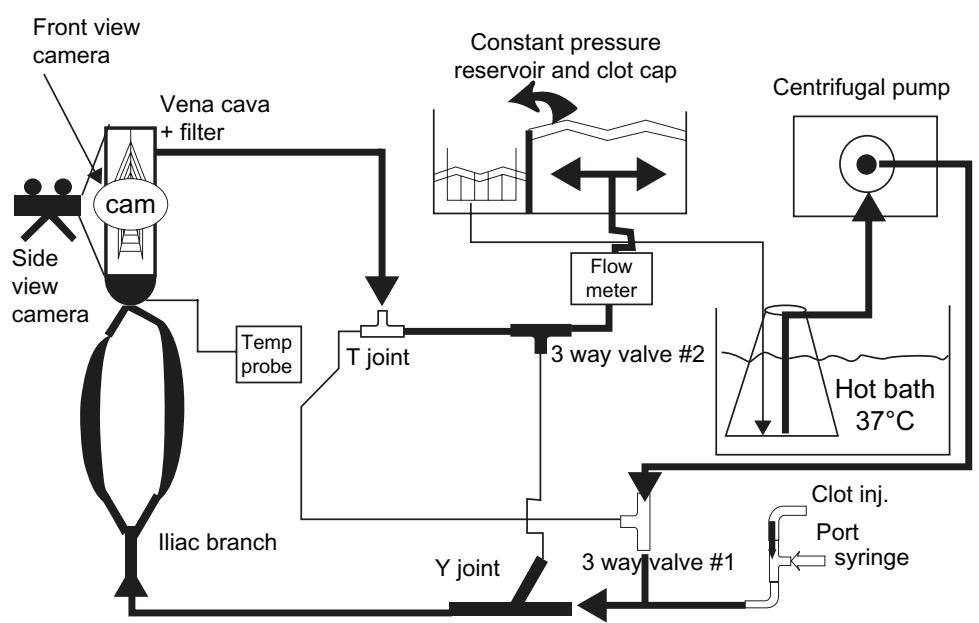

Figure I Schematic depicting the inferior vena cava flow loop in its forward flow configuration. Abbreviations: Cam, camera; clot cap, clot capture; Clot inj, clot injection; fwd, forward.

approximate human blood in the body. A centrifugal blood pump generated steady flow rates for the 20 and $28 \mathrm{~mm}$ diameter vena cava models of $1.0 \mathrm{~L} / \mathrm{min}$ (Reynolds number, $\mathrm{Re}=340)$ and $2.0 \mathrm{~L} / \mathrm{min}(\mathrm{Re}=490)$, respectively; they spanned a range of physiological, yet non-pulsatile, laminar flows. ${ }^{13}$ The silicone vena cava model was housed within an acrylic chamber filled with the glycerin-water solution for refractive index matching, which allowed for non-distorted observation of the clots.

\section{Preparation of polyacrylamide clots}

The moderately stiff PA hydrogel clots were made with acrylamide solutions obtained from Bio-Rad Laboratories (Hercules, CA, USA). They were prepared from $21 \mathrm{~mL}$ of $40 \%$ acrylamide stock solution, $14.4 \mathrm{~mL}$ of $2 \%$ bisacrylamide fixer solution, $203 \mathrm{~mL}$ distilled water, $1.2 \mathrm{~mL}$ of $10 \%$ ammonium persulfate, and $0.14 \mathrm{~mL}$ of tetramethylethylenediamine for a total volume of $240 \mathrm{~mL} .{ }^{14}$ The hydrogels were cast in long polyvinylchloride tubing with inner diameters of $3.2 \mathrm{~mm}, 4.8 \mathrm{~mm}$, and $6.4 \mathrm{~mm}$. Once polymerized, they were cut to the appropriate length (Figure 2A) and immersed in $0.1 \mathrm{~g} / \mathrm{dL}$ bovine serum albumin for 24 hours. Next, the $3.5 \%$ acrylamide $/ 0.12 \%$ bisacrylamide hydrogels were stained with Coomassie brilliant blue dye, which bound to the albumin to enhance optical visualization. The PA clot was washed with de-ionized water to remove any neurotoxic acrylamide monomers and methanol from the dye, and then soaked in a $1.12 \mathrm{~g} / \mathrm{cc}$ density glycerin-water solution over a 2-day period. PA hydrogels often swell and change density when placed in this media for an extended period of time. ${ }^{15}$ Thus a total preparation time of 5 days was required before the hydrogel clots were ready for testing. The PA hydrogels had a slightly higher density $(1.14 \mathrm{~g} / \mathrm{cc})$ than the glycerinwater blood analog $(1.12 \mathrm{~g} / \mathrm{cc})$. Nine different clot sizes were studied (Figure 2A), with diameters of 3.2, 4.8, and $6.4 \mathrm{~mm}$. The clot diameters varied by as much as $0.2 \mathrm{~mm}$ from the target value. If the clots were allowed to soak in the test fluid for an extended time ( $>24$ hours), then swelling could occur by $\sim 25 \%$. For each diameter, clots with lengthto-diameter ratios of 1:1,3:1, and 10:1 were cut. Soft and stiff PA clots with final concentrations of $3 \%$ acrylamide $/ 0.085 \%$ bisacrylamide and $4 \%$ acrylamide/ $0.16 \%$ bisacrylamide, respectively, were also formulated in a similar manner for comparative purposes..$^{14}$

\section{Preparation of sheep and human blood clots}

More than 30 animal blood clots were created in the same sizes as the medium and large long hydrogel clots to study actual blood clot characteristics and make a direct comparison to the synthetic clots (see Figure 2B). Human blood samples were collected from healthy volunteers using an approved clinical

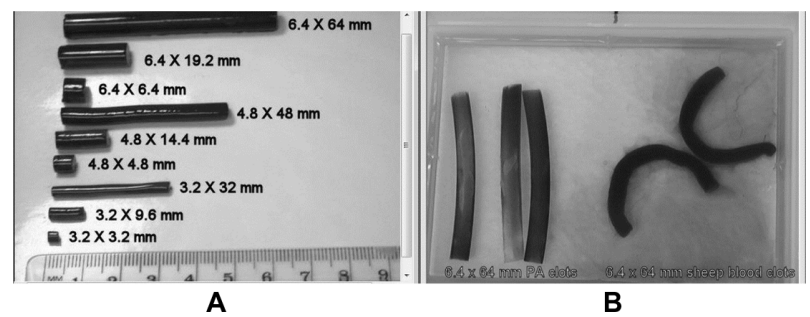

Figure 2 (A) All polyacrylamide (PA) clots used in original experiment, and (B, left) stiff, straight PA clots compared to (B, right) folding sheep blood clots. 
research protocol. Donor sheep blood (Lampire Biological Laboratories, AAALAC accredited, Pipersville, PA, USA), collected under animal use protocols approved by an Institutional Animal Care and Use Committee, and human blood were both anticoagulated with acid citrate dextrose solution A (1:8 ratio with blood). The hematocrits for the sheep and human blood were $31 \% \pm 2 \%$ and $41 \% \pm 3 \%$, respectively, but otherwise the physical properties of human and sheep blood clots were quite similar. Clotting was initiated by the addition of calcium chloride ( $20 \mathrm{mM}$ in blood), which acts as a co-factor in the coagulation process (intrinsic pathway) by counteracting the citrate. The blood was allowed to clot for at least 2 hours at $37^{\circ} \mathrm{C}$ before testing. The physical properties of human blood clots were measured, but the human blood clots were not studied in the mock flow loop.

\section{Measurement of dynamic elastic modulus of clots}

The components of the dynamic elastic modulus ( $\mathrm{E}^{\prime}$ and $\mathrm{E}^{\prime \prime}$ ) of the viscoelastic test clot materials were measured using an RSA-3 Dynamic Mechanical Analysis (DMA) compression tester (TA Instruments, New Castle, DE, USA). To optimize the sensitivity, reliability, and reproducibility of the elasticity measurements, large cylindrical clot plugs with diameters of $25 \mathrm{~mm}$ and thicknesses ranging from 10 to $15 \mathrm{~mm}$ were created using both blood and PA hydrogels. After the clot material was secured between two parallel compression plates, the exposed surfaces of the clots were wetted with saline or oil to reduce surface tension and minimize exposure of the sample to air. A compression force of $1.5 \pm 0.5 \mathrm{~N}$ was initially applied to the clot to hold it in place.

Strain and time sweep tests were conducted in compression mode to characterize clot elasticity. First, a strain sweep covering a strain range of $0.5 \%-10 \%$ was used to determine the region where storage modulus was independent of strain. In most cases, an optimal strain of $1 \%$ and a $1 \mathrm{~Hz}$ frequency were used to make oscillating compression measurements. Next, a time sweep was performed by applying a constant $1 \%$ strain for up to 10 minutes at the same $1 \mathrm{~Hz}$ frequency. The dynamic elastic modulus (Equation 2) was determined from the slope of the stress-strain curve.

\section{Statistical analysis}

Binomial sampling theorem was performed on all of the clot injection experiments (500-580 for each filter). Using binomial sampling theory, for each test condition, if the first 10 clots were captured, then that condition was considered to have $100 \%$ capture efficiency. If at least one clot passed through the filter, an additional 10 experiments were conducted at that test condition. ${ }^{12}$ If, after 20 clot injection experiments, the CCE was below $90 \%$, an additional 10 experiments were performed. At most, a total of 30 dynamic capture experiments were performed at any one condition. After collecting the data, the Student $t$-test and binary logistic regression analyses were performed to evaluate the effect of different experimental variables (eg, filter type, vena cava diameter, vena cava orientation, clot diameter, and clot length) on the filters' clot capture efficiency.

\section{Results}

Representative clot capture efficiency results are presented in Table 1 for the six IVC filter designs. Three of the nine PA clot sizes in the $28 \mathrm{~mm}$ diameter IVC model, with a $2 \mathrm{~L} / \mathrm{min}$ flow-rate in both the horizontal and vertical test orientation, are shown here. Filter 1 had the lowest clot capture efficiencies for the larger hydrogel clot sizes, when mounted horizontally in the large diameter IVC model. The clot capture efficiencies for the $4.8 \times 48 \mathrm{~mm}$ and $6.4 \times 64 \mathrm{~mm}$ hydrogel clots were $40 \%$ and $23 \%$, respectively, compared with $67 \%-100 \%$ capture efficiencies for all other filters. Overall, the PA clot capture efficiency was similar across all filters for the remaining hydrogel sizes, vessel size, flow

Table I Clot capture efficiencies (\%) for the six tested filter designs $(n=10-30)$ for the I0:I length: diameter PA clots in the 28 mm diameter IVC filter at $2 \mathrm{~L} / \mathrm{min}$

\begin{tabular}{llllllllll}
\hline Material & Clot diameter & Clot length & Orientation & Filter I & Filter 2 & Filter 3 & Filter 4 & Filter 5 & Filter 6 \\
\hline PA & $3.2 \mathrm{~mm}$ & $32 \mathrm{~mm}$ & Horizontal & 90 & 100 & 77 & 67 & 73 & 90 \\
PA & $4.8 \mathrm{~mm}$ & $48 \mathrm{~mm}$ & Horizontal & $\mathbf{4 0}$ & 100 & 85 & 85 & 70 & 95 \\
PA & $6.4 \mathrm{~mm}$ & $64 \mathrm{~mm}$ & Horizontal & $\mathbf{2 3}$ & 100 & 100 & 95 & 85 & 100 \\
PA & $3.2 \mathrm{~mm}$ & $32 \mathrm{~mm}$ & Vertical & 100 & 100 & 85 & 85 & 85 & 100 \\
PA & $4.8 \mathrm{~mm}$ & $48 \mathrm{~mm}$ & Vertical & 100 & 100 & 90 & 90 & 100 & 100 \\
PA & $6.4 \mathrm{~mm}$ & $64 \mathrm{~mm}$ & Vertical & 100 & 100 & 100 & 100 & 100 & 100 \\
Sheep blood & $4.8 \mathrm{~mm}$ & $48 \mathrm{~mm}$ & Horizontal & 95 & - & - & - & - & - \\
Sheep blood & $6.4 \mathrm{~mm}$ & $64 \mathrm{~mm}$ & Horizontal & 100 & - & - & - & - & - \\
\hline
\end{tabular}

Notes: Here, the $\mathrm{E}^{\prime}$ of polyacrylamide (PA) was estimated ${ }^{14}$ to be $\sim 1800 \mathrm{~Pa}$, and the $\mathrm{E}^{\prime}$ of the sheep blood clots was measured to be $\sim 1700 \mathrm{~Pa}$. Data for $4.8 \times 48 \mathrm{~mm}$ and $6.4 \times 64 \mathrm{~mm}$ sheep blood clots with Filter I are also shown. The bolded values are the two observed anomalies (low clot capture efficiency) for Filter I.

Abbreviation: IVC; inferior vena cava. 
rates, and orientation for the large $28 \mathrm{~mm}$ IVC model. Clot capture efficiency was assessed as a function of filter type, vena cava diameter ( small $=20 \mathrm{~mm}$ and large $=28 \mathrm{~mm}$ ), vena cava orientation (horizontal or vertical), and clot diameter and length. Clots were more likely to be captured in the small vena cava than in the large one $(P<0.0001)$, and with the vena cava model in the vertical orientation rather than the horizontal configuration $(P<0.0001)$. In general, large and medium diameter clots were more likely to be captured than small diameter clots $(P<0.0001)$.

In contrast to the $23 \%$ and $40 \%$ clot capture efficiencies of the 6.4 and $4.8 \mathrm{~mm}$ diameter synthetic hydrogel clots, respectively, in Filter 1, the same filter produced capture rates of $95 \%-100 \%$ for similarly-sized sheep blood clots. None of the other test filters generated low $(<70 \%)$ clot capture efficiencies for the PA clots using the same configuration. The physical characteristics of the surrogate clot, not the filter itself, are suspected as the cause of this anomaly. Lastly, as expected, the clot capture efficiency was low $(21 \%-54 \%)$ for the smaller, less dangerous clots regardless of material composition or filter design.

The clot dynamics of the synthetic hydrogel clots were noticeably different from those of the sheep blood clots. Figure 3A depicts how moderately stiff PA clots flowed from right to left through the loop without tumbling. It was much more difficult for certain filters to capture these cylindrical clots due to their relatively small exposed cross-sectional area. In contrast, the sheep blood clots and soft PA clots

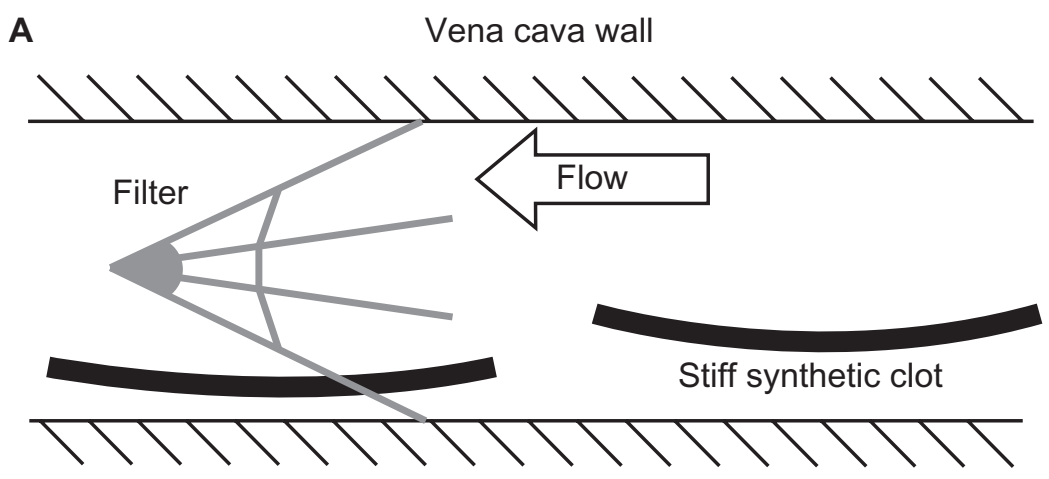

B
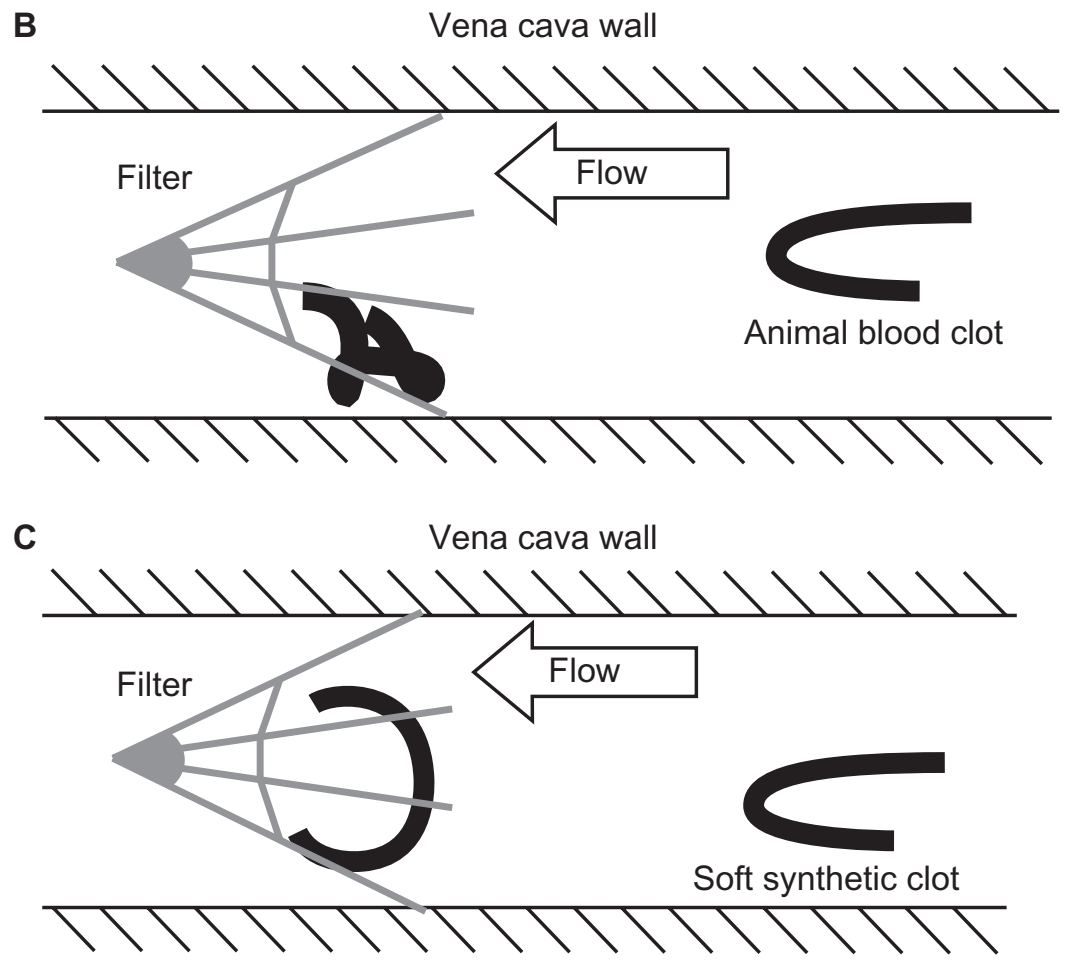

Figure 3 Illustrations of a typical clot configuration within the flow field and interaction with the filter. (A) Stiff polyacrylamide clot flowing toward and passing through a generic inferior vena cava filter. (B) Folded animal blood clot approaching the filter with capture by the filter. (C) Soft, 800 Pa polyacrylamide clot captured by the filter but with less convoluted folding. 
were more flexible and folded as they approached the filter (Figure 3B and C). Once captured, these clots would wrap around the wire struts of the filter. The surface imperfections on the sheep blood clots may have promoted the observed folding behavior (Figures 2B and 3B).

After soaking in the glycerin-water solution for 2 days, the largest synthetic PA clots, which were slightly more dense $(1.12 \mathrm{~g} / \mathrm{cc})$ than the test fluid $(1.10 \mathrm{~g} / \mathrm{cc})$, tended to flow along the bottom of the horizontal vena cava with their long axis parallel to the vena cava walls. However, smaller PA clots were more buoyant and tended to flow in the center of the lumen. Hydrogel clots were more likely to pass through the filter when it was positioned such that a gap in the peripheral filter wires was located along the lower portion of the cava wall, as shown in Figure 4. When a peripheral filter strut happens to extend toward the bottom of the vessel, the PA was more likely to be captured. The sheep blood clots also tended to stay near the bottom of the simulated IVC when in the horizontal orientation, thus demonstrating similar buoyancy effects as the synthetic clots. However, in contrast to the synthetic PA clots, the more flexible sheep blood clots folded in all cases as they passed through the disturbed flow region of the iliac bifurcation located directly before the IVC. The folding behavior of the sheep blood clots resulted in an effectively larger exposed cross-sectional area when passing through the filter, increasing their likelihood of capture. As expected, the capture rate increased for larger clot sizes for the same reason. ${ }^{16}$

The marked difference in the deformability of the sheep blood clots and PA hydrogel clots under flow conditions prompted a more careful investigation of the role of dynamic elastic modulus. The measured $\mathrm{E}^{\prime}$ of human, sheep, and

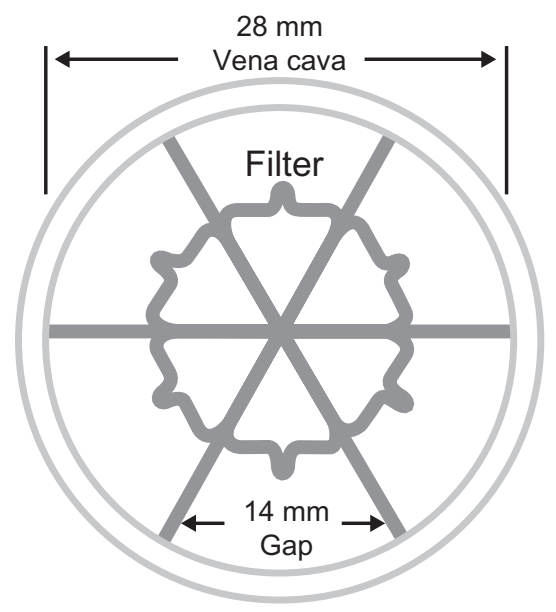

Figure 4 The inferior vena cava filter positioned such that a gap existed along the dependent side of the vena cava was less likely to capture the polyacrylamide clot. three synthetic clot formulations are given in Table 2. The $\mathrm{E}^{\prime}$ of the sheep blood clots $(1690 \pm 360 \mathrm{~Pa})$ was similar to that of the moderately stiff clots (1696 $\pm 10 \mathrm{~Pa})$. Although $\mathrm{E}^{\prime}$ values for the two were similar, the moderately stiff PA clots failed to replicate the folding behavior of the sheep blood clots. The elastic modulus of the soft PA clots $\left(\mathrm{E}^{\prime}=805 \pm 2 \mathrm{~Pa}\right)$ matches the value reported by Jaeger in his studies with PA clots. ${ }^{9}{ }^{10}$ Moreover, the soft PA clot most closely matched the flow loop deformation behavior of the sheep blood clots, especially compared with the moderately stiff and stiff PA clots (1696 $\pm 10 \mathrm{~Pa}$ and $3295 \pm 37 \mathrm{~Pa}$, respectively). The magnitude of the loss modulus ( $\mathrm{E}^{\prime \prime}$ ) was small at $<2 \%$ and $<15 \%$ of the storage modulus ( $E^{\prime}$ ) for the PA hydrogels and blood clots, respectively, which signified that both clot types behaved more like solids than fluids. Although human blood clot capture was not studied in our IVC flow loop, the storage elastic modulus values for human blood clots were slightly lower than for sheep blood clots (Table 2).

\section{Discussion}

In the flow loop experiments, one filter design yielded significantly lower clot capture efficiencies compared to the other IVC filters for the largest synthetic clot sizes with the $28 \mathrm{~mm}$ IVC vessel in the horizontal position. PA hydrogel clots behaved differently than the sheep blood clots at this test condition. While some of the hydrogel clot capture efficiencies were low, the results with sheep blood clots were higher (>95\%) and comparable to the similarly sized hydrogel clot capture data for the other filters in this test configuration. In general, the sheep blood clots $\left(\mathrm{E}^{\prime}=1690 \pm 360 \mathrm{~Pa}\right)$ folded, whereas the moderately stiff PA hydrogels with a comparable value of $E^{\prime}(1696 \pm 10 \mathrm{~Pa})$

Table 2 Comparison between measurements of components of dynamic elastic modulus ( $E^{\prime}$ and $E^{\prime \prime}$ ) of polyacrylamide synthetic clots and blood clots made from sheep and human blood

\begin{tabular}{lccl}
\hline & $E^{\prime}$ (pascals) & $E^{\prime \prime}$ (pascals) & $\begin{array}{l}\text { Behavior in } \\
\text { flow loop }\end{array}$ \\
\hline $\begin{array}{l}\text { Sheep blood }(\mathrm{n}=3) \\
\text { Human blood }(\mathrm{n}=3)\end{array}$ & $1690 \pm 360$ & $187 \pm 8$ & $\begin{array}{l}\text { Folds } \\
\text { (Not studied) }\end{array}$ \\
$\begin{array}{l}\text { Soft polyacrylamide } \\
(3 \% \text { acryl/0.085\% bis) }\end{array}$ & $805 \pm 2$ & $12 \pm 1$ & Folds \\
$\begin{array}{l}\text { Moderately stiff } \\
\text { polyacrylamide }\end{array}$ & $1696 \pm 10$ & $26 \pm 1.2$ & Does not fold \\
$\begin{array}{l}3.5 \% \text { acryl/0.12\% bis) } \\
(\mathrm{n}=3)\end{array}$ & & & \\
$\begin{array}{l}\text { Stiff polyacrylamide } \\
(4 \% \text { acryl/0.16\% bis) }\end{array}$ & $3295 \pm 37$ & $16 \pm 8$ & Does not fold \\
\hline
\end{tabular}

Abbreviations: acryl, acrylamide; bis, bisacrylamide. 
tended to maintain their unperturbed shapes flowing lengthwise along the bottom of the IVC lumen. Only by using soft hydrogel synthetic clots $\left(E^{\prime}=805 \pm 2 \mathrm{~Pa}\right)$ were we able to replicate the flow loop behavior of the sheep blood clots. In addition to clot properties, filter structure can contribute to the local flow dynamics of the clots and the capture efficiency of the stiffer PA clots. In these studies, when oriented such that one of its struts extended to the lower portion of the lumen, the filter tended to capture the large clots. Other literature reports of in vitro studies have shown influences of orientation (horizontal or vertical) and filter position (concentric or eccentric) on clot capture efficiency. ${ }^{16}$ Ideally, a filter should be effective regardless of its position or orientation, since those factors could be constantly changing with body position in a mobile patient and are not necessarily dictated during the implant procedure. Sheep blood clot capture was independent of filter orientation, again demonstrating that the observed CCE anomaly was due to the lack of buoyancy and stiffness of the PA clots used, and not the filter design or filter orientation. While these flow studies focused on clot capture efficiency of an empty filter, the literature suggests that previously captured clots in a filter will further affect the local flow fields through IVC filters. ${ }^{17}$

In terms of clot structure, the PA hydrogel clots were homogeneous, which is not the case with blood clots. During the formation of fibrin networks, plasma and other blood components may be excluded from the clot, leading to a non-homogenous structure. The inconsistent shape of blood clots may explain why they tend to fold more easily than the completely cylindrical hydrogel clots despite having similar elastic modulus values and being subjected to similar fluid stresses. Furthermore, the DMA instrument was only able to make accurate elastic modulus measurements on short cylindrical plug materials $(25 \mathrm{~mm}$ diameter, with a $10-15 \mathrm{~mm}$ thickness) and not on the actual clot sizes $(6.4 \mathrm{~mm}$ diameter and $64 \mathrm{~mm}$ long) used in the dynamic testing loop. The relatively large sample volume for the compression testing may also have masked important effects of sample clot heterogeneity. Also, polyacrylamide clots may become stiffer with age, due to changes in cross-linking over time. ${ }^{18}$ Because the properties of both clot types are subject to change over time, only freshly-made clots ( $<24$ hours old) should be used.

In general, the reported physical parameters for clots will largely depend on the method of measurement (ie, local-scale versus bulk properties) and characteristics of the clot (eg, clot age, volume of the clot, clot shape and homogeneity, patient
Table 3 Elastic modulus of human whole blood clots in the literature

\begin{tabular}{|c|c|c|c|}
\hline Reference & $\begin{array}{l}\text { Elastic } \\
\text { modulus } \\
\text { (pascals) }\end{array}$ & $\begin{array}{l}\text { Shear } \\
\text { modulus } \\
\text { (pascals) }\end{array}$ & $\begin{array}{l}\text { Method of } \\
\text { measurement }\end{array}$ \\
\hline Schmitt et $\mathrm{al}^{20}$ & $930 \pm 189 *$ & $310 \pm 63$ & $\begin{array}{l}\text { Cone and plate } \\
\text { rheometer }\end{array}$ \\
\hline Dempfle et $a^{21}$ & $\sim 2100$ & - & $\begin{array}{l}\text { Compression } \\
\text { testing }\end{array}$ \\
\hline Brophy et $\mathrm{al}^{22}$ & $1380 \pm 920$ & - & $\begin{array}{l}\text { Compression } \\
\text { testing }\end{array}$ \\
\hline Carr et $\mathrm{a}^{23}$ & $2318 \pm 74$ & - & $\begin{array}{l}\text { Cone and plate } \\
\text { rheometry }\end{array}$ \\
\hline Diamond ${ }^{24}$ & $900 *$ & $\sim 300$ & Not specified \\
\hline Whitbourne $e^{25}$ & $934 \pm 100$ & - & $\begin{array}{l}\text { Thrombo } \\
\text { viscoelastography }\end{array}$ \\
\hline Isogai et $\mathrm{al}^{26}$ & $339 \pm 76$ & - & Rheometry \\
\hline
\end{tabular}

Note: *Estimated elastic modulus under the assumption that $v=0.495 .{ }^{27}$

disease, and method of clot formation). ${ }^{3,19,20}$ Table 3 shows measurements of the elastic modulus and shear modulus values for human blood clots reported in the literature, along with the corresponding measurement methods used in each study. As expected, the reported elastic modulus of blood clots varies widely in the literature, from approximately $340-2320 \mathrm{~Pa},{ }^{20-26}$ thus it is not obvious which elastic modulus value should be matched when testing an IVC filter with synthetic clots. Whatever elastic modulus value is selected for the synthetic clot, the fluid dynamics of the synthetic clot should be similar to an animal or human blood clot in an in vitro flow loop, to avoid the unexpected results presented in this paper. Local-scale measurements such as atomic force microscopy or micro-indentation methods are more dependent on the surface properties of the material than those methods which utilize bulk compression measurements that deform the entire clot and better assess the three-dimensional integrity of its structure. Each elasticity measurement technique has different limitations and underlying assumptions, which have resulted in large discrepancies in the reported elastic modulus values. In particular, the assumption that the material is homogeneous and isotropic may not be appropriate. Moreover, there is no standard measurement material available for comparing the elastic modulus of soft hydrogels between instruments.

To further investigate the anomalies observed in our original clot capture study using PA hydrogel clots in one IVC filter, we evaluated the elastic modulus and flow characteristics of sheep blood clots and PA hydrogel clots over a large stiffness range. Material characteristics (eg, elastic modulus, clot shape, and clot density) of synthetic clots should match those made on actual blood clots using 
the same measurement equipment and conditions. However, the elastic modulus may not be the only important property, as the surface imperfections of the actual blood clots likely contribute to the observed folding behavior in the flow loop. The best match to the behavior of sheep blood clots was achieved using the soft PA clots having a lower elastic modulus $\left(\mathrm{E}^{\prime}=805 \pm 2 \mathrm{~Pa}\right)$. The elastic modulus is a simplified characterization of the complex material properties of synthetic hydrogels and blood clots, which may exhibit different nonlinear stress-strain responses such as anisotropy and non-homogeneity; this may explain why similar overall behavior was found in samples with different elastic modules (ie, sheep blood clots $\mathrm{E}^{\prime}=1690 \pm 360 \mathrm{~Pa}$, soft PA clots $\mathrm{E}^{\prime}=805 \pm 2 \mathrm{~Pa}$ ). Thus, the behavior of the clot within the loop should accurately represent that of a physiological clot.

\section{Conclusion}

In summary, although synthetic clots may be more convenient and easier to use than real blood clots and their properties can be easily adjusted based on formulation, these data demonstrate limitations in using such surrogate clots for determining clot capture efficiency of an IVC filter. In this study, differences in the physical characteristics of the clot material, such as shape, density, and elasticity, had an impact on the measured filter efficiency. However, the clot composition had a negative effect on capture efficiency in only one filter in the horizontal position that had gaps in its peripheral wire struts positioned along the lower vena cava wall. Clot dynamics within an in vitro flow loop donot solely depend on the measured elastic modulus. The overall flow behavior of a clot within the loop is a more important parameter for assessing clot capture efficiency of IVC filters.

If animal or human blood clots of appropriate size cannot be used to perform the in vitro IVC filter clot capture efficiency experiments, polyacrylamide hydrogels may be used as surrogates if they have similar elastic modulus and demonstrate similar folding flow loop characteristics. However, a careful assessment of certain characteristics such as flow behavior and elastic modulus of hydrogel clots compared to clinically relevant blood clots should be performed to properly validate their use, and fresh clots ( $<24$ hours), regardless of material, should be used to study the CCE of IVC filters.

\section{Disclaimer}

The mention of commercial products, their source, or their use in connection with the material reported herein, is not to be construed as either an actual or implied endorsement by the
US Food and Drug Administration, the Department of Health and Human Services, or the Public Health Service.

\section{Acknowledgments}

The authors thank Shiva Yazdani, who helped perform the clot capture efficiency tests and analyzed a large portion of the clot capture data, Dr Maureen Dreher for technical consultation, Dr Harry Bushar for assisting with the statistical analysis, and the Research Blood Donor program at the National Institutes of Health Blood Bank.

\section{Disclosure}

The authors have no conflicts of interest to report in this work.

\section{References}

1. Guidance for Cardiovascular Intravascular Filter $510(\mathrm{k})$ Submissions, November 1999. Available from: www.fda.gov/MedicalDevices/ DeviceRegulationandGuidance/GuidanceDocuments/ucm073776.htm. US Food and Drug Administration. Accessed April 24, 2013.

2. Streiff MB. Vena cava filters: a comprehensive review. Blood. 2000; 95:3669-3677.

3. Smith AC, Abel DB. Regulation of peripheral vascular devices. Endovascular Today. 2005:93-94.

4. Simon M, Rabkin BS, Kleshinski BS, et al. Comparative evaluation of clinically available inferior vena cava filters with an in vitro physiological simulation of the vena cava. Cardiovasc Radiol. 1993;189: 769-774.

5. Mahnken AH, Pfeffer J, Stanzel S, Mossdorf A, Günther RW, Schmitz-Rode T. In vitro evaluation of optionally retrievable and permanent IVC filters. Invest Radiol. 2007;42:529-535.

6. Stecker M, Barnhart WH, Lang EV. Evaluation of a spiral nitinol temporary inferior vena cava filter. Acad Radiol. 2001;8:484-493.

7. Greenfield LJ, Proctor MC. Experimental embolic capture by asymmetric Greenfield filters. J Vasc Surgery. 1992;16:436-444.

8. Proctor MC, Cho KJ, Greenfield LJ. Development and evaluation of investigational vena cava filters: the complementary roles of in vitro and in vivo studies. J Surg Res. 2003;10:241-254.

9. Jaeger HJ, Mair T, Geller M, et al. A physiological in vitro model of the inferior vena cava with a computer-controlled flow system for testing of inferior vena cava filters. Invest Radiol. 1997;32:511-522.

10. Jaeger HJ, Kolb S, Mair T, et al. In vitro model for the evaluation of inferior vena cava filters: effect of experimental parameters on thrombus-capturing efficacy of the vena tech LGM filter. JVasc Interv Radiol. 1998;9:295-304.

11. Malinauskas RA, Sarraf P, Barber KM, Truskey GA. Association between secondary flow in models of the aorta-celiac junction and sub endothelial macrophages in the normal rabbit. Atherosclerosis. 1998; 140:121-134.

12. Comulda WS, Weiss RE. On models for binomial data with random numbers of trials. Biometrics. 2007;63:610-617.

13. Cheng CP, Herftkens RJ, Taylor CA. Inferior vena cava hemodynamics quantified in vivo at rest and during exercise using magnetic resonance imaging. Am J Physiol Heart Circ Physiol. 2002;284:H1161-H1167.

14. Tse JR, Engler AJ. Preparation of hydrogel substrates with tunable mechanical properties. Curr Protoc Cell Biol. 2010;10:16.1-16.16.

15. Baker BA, Murff RL, Milam VT. Tailoring the mechanical properties of polyacrylamide-based hydrogels. Polymer. 2010;51:2207-2214.

16. Lorch H, Dallmann A, Zwaan M, Weiss HD. Efficacy of permanent and retrievable vena cava filters: experimental studies and evaluation of a new device. Cardiovasc Intervent Radiol. 2002;25:193-199. 
17. Stewart SFC, Robinson RA, Nelson RA, Malinauskas RA. Effects of thrombosed vena cava filters on blood flow: flow visualization and numerical modeling. Ann Biomed Eng. 2008;36: 1764-1781.

18. Ilavsky M, Hrouz J, Stejskal J, Bouchal K, et al. Phase transition in swollen gels: effect of aging on the extent of hydrolysis of aqueous polyacrylamide solutions and on the collapse of gels. Macromolecules. 1984; 17:2868-2874.

19. Riha P, Wang X, Liao R, Stoltz JF. Elasticity and fracture strain of whole blood clots. Clin Hemorheol and Microcirc. 1999;21:45-49.

20. Schmitt C, Henni AH, Cloutier G. Characterization of blood clot viscoelasticity by dynamic ultrasound elastography and modeling of the rheological behavior. J Biomech. 2011;44:622-629.

21. Dempfle CE, Kalsch T, Elmas E, et al. Impact of fibrinogen concentration in severely ill patients on mechanical properties of whole blood clots. Blood Coagul Fibrinolysis. 2008;19:765-770.
22. Brophy DF, Martin RJ, Gehr TWB, Carr ME. A hypothesis-generating study to evaluate platelet activity in diabetics with chronic kidney disease. Thrombosis. 2005;3:1-6.

23. Carr ME, Krishnaswami A, Martin EJ. Platelet contractile force (PCF) and clot elastic modulus (CEM) are elevated in diabetic patients with chest pain. Diabet Med. 2002;19:862-866.

24. Diamond SL. Engineering design of optimal strategies for blood clot dissolution. Annu Rev Biomed Eng. 1999;1:427-461.

25. Whitbourne PGS. Changes in the Clotting Viscoelasticity Caused by Cardiopulmonary Bypass (CPB) Surgery [dissertation]. Cambridge, MA: Massachusetts Institute of Technology; 1998.

26. Isogai Y, Iida A, Chikatsu I. Dynamic viscoelasticity of blood during clotting in health and disease. Biorheol. 1973;10:411-424.

27. Chippada U, Yurke B, Langrana NA, et al. Simultaneous determination of Young's Modulus, shear modulus and Poisson ratio of soft hydrogels. $J$ Mater Res. 2010; 25:545-555.
Medical Devices: Evidence and Research

\section{Publish your work in this journal}

Medical Devices: Evidence and Research is an international, peerreviewed, open access journal that focuses on the evidence, technology, research, and expert opinion supporting the use and application of medical devices in the diagnosis, treatment and management of clinical conditions and physiological processes. The identification of novel

\section{Dovepress}

devices and optimal use of existing devices which will lead to improved clinical outcomes and more effective patient management and safety is a key feature. The manuscript management system is completely online and includes a quick and fair peer-review system. Visit http://www. dovepress.com/testimonials.php to read real quotes from authors.

\footnotetext{
Submit your manuscript here: http://www.dovepress.com/medical-devices-evidence-and-research-journal
} 